\title{
Preparation and in vivo pharmacokinetics of curcumin-loaded PCL-PEG-PCL triblock copolymeric nanoparticles
}

This article was published in the following Dove Press journal:

International Journal of Nanomedicine

26 July 2012

Number of times this article has been viewed

\section{Runliang Fengl,* \\ Zhimei Songl,* \\ Guangxi Zhai ${ }^{2}$}

'Department of Pharmaceutical Engineering, College of Medicine and Life Science, University of Jinan, Jinan, Shandong Province, ${ }^{2}$ Department of Pharmaceutics, College of Pharmacy, Shandong University, Jinan, Shandong Province, People's Republic of China

*These authors contributed equally to this work
Correspondence: Runliang Feng Department of Pharmaceutical Engineering, College of Medicine and Life Science, University of Jinan, 106 Jiwei Road, Jinan 250022, Shandong Province, People's Republic of China Tel +8653189736825

Fax +8653189736818

Email runliangfeng@yahoo.cn
Background: Curcumin (CUR) has been linked with antioxidant, anti-inflammatory, antimicrobial, anti amyloid, and antitumor effects, but its application is limited because of its low aqueous solubility and poor oral bioavailability.

Methods: To improve its bioavailability and water solubility, we synthesized two series of poly ( $\varepsilon$-Caprolactone)-poly (ethylene glycol)-poly ( $\varepsilon$-Caprolactone) triblock copolymers by ring-opening polymerization of poly (ethylene glycol) and $\varepsilon$-Caprolactone, with stannous 2-ethylhexanoate as the catalyst. Structure of the copolymers was characterized by proton nuclear magnetic resonance spectroscopy, Fourier transform infrared spectroscopy, and gel permeation chromatography. The nanoparticles (NPs) were prepared using a probe-type ultrasonic emulsion and solvent evaporation method. To obtain an optimal delivery system, we explored the effect of the length of the copolymers' hydrophilic and hydrophobic chains on the encapsulation of hydrophobic CUR, performing entrapment efficiency and drug loading evaluations, as well as studying the particle distribution and in vitro release using the direct dispersion method. Finally, study of the in vivo pharmacokinetics of the CUR-loaded NPs was also carried out on selected copolymers in comparison with CUR solution formulations.

Results: CUR was encapsulated with $94.3 \%$ and $95.5 \%$ efficiency in biodegradable nanoparticulate formulations based on NP43 and NP63, respectively. Dynamic laser light scattering and transmission electron microscopy indicated a particle diameter of $55.6 \mathrm{~nm}$ and $62.4 \mathrm{~nm}$ for NP43 and NP63, respectively. Fourier transform infrared spectroscopy and differential scanning calorimetry analysis of the NPs showed that CUR was encapsulated into the NPs. The in vitro release experiments showed that NP63 controlled the release of CUR more effectively, with only $55 \%$ of CUR released after 96 hours. In comparison with the free-drug solution in vivo, encapsulation of the CUR in NP63 increased mean residence time from 0.169 to 40.148 hours and the area under the concentration-time curve 4.178-fold.

Conclusion: CUR was effectively entrapped by the prepared NPs, which could improve the solubility of CUR and prolong its retention in the systemic circulation.

Keywords: nanoparticle, copolymer, solubility, hydrophilic chain, hydrophobic chain

\section{Introduction}

Curcumin (CUR) (Figure 1) is a low molecular weight, natural polyphenolic compound that is isolated from the rhizome of turmeric (Curcuma longa). It has a low intrinsic toxicity with a wide range of pharmacological activities, including antioxidant, antiinflammatory, antimicrobial, anti amyloid, and antitumor properties. ${ }^{1,2}$ Preclinical studies of CUR have shown its ability to inhibit carcinogenesis in a variety of cell lines. ${ }^{3}$ The ability of CUR to induce apoptosis in cancer cells without cytotoxic effects on healthy cells makes it a potential compound for drug development against cancer. ${ }^{3,4}$ 


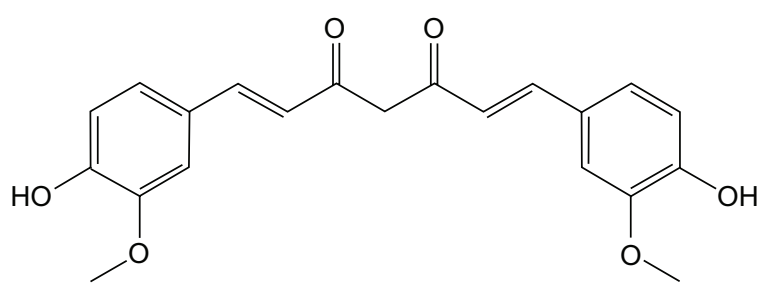

Figure I Structure of curcumin.

However, its clinical potential has been greatly limited due to extremely low solubility in aqueous solution $\left(2.99 \times 10^{-8} \mathrm{M}\right)$ as well as its poor bioavailability. ${ }^{5,6}$

Copolymeric nanoparticles (NPs) have received a lot of attention due to their stability and ease with which their surfaces can be modified. ${ }^{7}$ They can be tailor-made to achieve both controlled drug release and disease-specific localization by tuning the polymer characteristics and surface chemistry. ${ }^{8-12}$ A study has shown that nanocarriers can become concentrated preferentially in tumors, at inflammatory sites, and at antigen-sampling sites by virtue of the enhanced permeability and retention effect of the vasculature. ${ }^{13}$ Once accumulated at the target site, hydrophobic biodegradable polymeric NPs can act as a local drug depot, providing a source for a continuous supply of encapsulated therapeutic compound(s) at the disease site (such as at solid tumors).

In this study, biodegradable poly ( $\varepsilon$-Caprolactone)-poly (ethylene glycol)-poly ( $\varepsilon$-Caprolactone) (PCL-PEG-PCL) copolymers were synthesized. CUR-NPs were produced from the self-assembly of the copolymer, assisted by a probe-type ultrasonic emulsion and solvent evaporation method without any surfactants. The CUR-NPs could be lyophilized into powder form. In addition, the characteristics of the NPs (such as their physico-chemical properties, in vitro release, and in vivo pharmacokinetics) were investigated in detail, for their application as a potential drug-delivery vehicle. CUR-NPs may have great clinical potential as a novel drugdelivery method.

\section{Materials and methods Materials}

CUR was purchased from Fluka Chemical Company Inc (Buchs, Switzerland). Poly (ethylene glycol) (PEG) $(\mathrm{Mn}=4000,6000)$ and stannous 2-ethylhexanoate $\left(\mathrm{Sn}(\mathrm{Oct})_{2}\right)$ were procured from Sigma Chemicals (St Louis, MO), while $\varepsilon$-Caprolactone $(\varepsilon-\mathrm{CL})$ was provided by Huayuan Polymer Co, Ltd (Qingtao, Shandong Province, China). All other chemicals and solvents were of analytical grade or higher, obtained commercially.

\section{Synthesis and characterization of PCL-PEG-PCL triblock copolymers}

The triblock copolymers were synthesized by ring-opening polymerization of different weights of $\varepsilon$-CL using PEG as a macro-initiator and $\mathrm{Sn}(\mathrm{Oct})_{2}$ (ca $0.1 \%$ of $\varepsilon$-CL in molar amount) as a catalyst. ${ }^{14} \mathrm{PEG}$ was added to a dry three-necked flask and heated at $120^{\circ} \mathrm{C}$ under vacuum for 3 hours to remove moisture. The $\varepsilon$-CL and $\mathrm{Sn}(\mathrm{Oct})_{2}$ were introduced into the flask under a nitrogen atmosphere and then the polymerization reaction was performed at $140^{\circ} \mathrm{C}$ with vigorous stirring for 8 hours. After cooling to room temperature, the mixture was dissolved in methylene dichloride and precipitated using cold ethyl ether for purification. Then the mixture was filtered and the purification process was repeated twice more. The obtained precipitate was vacuum-dried to a constant weight and stored in a desiccator until use.

The obtained copolymer was characterized by Fourier transform infrared spectroscopy (FT-IR) (potassium bromide) (Nicolet Nexus 470 ESP FT-IR, ThermoFisher Scientific, Waltham, MA), proton nuclear magnetic resonance ( ${ }^{1} \mathrm{H}-\mathrm{NMR}$ ) spectroscopy (in deuterated chloroform [CDCl${ }_{3}$ ) (AVANCE $300 \mathrm{MHz}$, Bruker, Germany), and gel permeation chromatography (GPC) (in chloroform $\left[\mathrm{CHCl}_{3}\right]$ ) (Agilent 110 HPLC, Santa Clara, CA).

\section{Preparation of CUR-free and -loaded NPs}

NPs were prepared using a probe-type ultrasonic emulsion and solvent evaporation method. ${ }^{15}$ To begin, $50 \mathrm{mg}$ of CUR and $350 \mathrm{mg}$ of PCL-PEG-PCL copolymer were dissolved in $20 \mathrm{~mL}$ of acetone. The solution was then introduced into $50 \mathrm{~mL}$ of water. The resulting mixture was subsequently sonicated 20 times using a probe-type sonicator (JY92-II, Scientz Biotechnology Co, Ltd, Ningbo, China) at $400 \mathrm{~W}$ for 6 minutes. The pulse was turned off for 5 seconds at 15 -second intervals and the oil in water emulsion formed. The acetone was then thoroughly removed by rotary vacuum evaporation at $35^{\circ} \mathrm{C}$. The resulting yellowish aqueous solution was filtered through a $0.45 \mu \mathrm{m}$ filter membrane to remove the unloaded CUR. Drugunloaded NPs were produced in a similar manner.

\section{Characterization of NPs' physico- chemical properties \\ Morphology of CUR-free and -loaded NPs}

Transmission electron microscopy (TEM) (JEM-1200EX, JEOL, Tokyo, Japan) was used for morphological observation. One drop of NP solution was placed on a carbon film-coated copper grid (400 mesh) followed by negative staining with 
$2 \%$ phosphotungstic acid for 20 seconds, absorption of excess solution with filter paper, and air drying, prior to placing it in the TEM instrument for analysis.

\section{Particle size and zeta potential of CUR-free and -loaded NPs}

Dynamic light scattering was used to measure the particle sizes of the CUR-free and -loaded NPs (Zetasizer 3000HS, Malvern Instruments Ltd, UK). Particle size was determined using a He-Ne laser beam of $633 \mathrm{~nm}$ wavelength with a fixed scattering angle of $90^{\circ}$ at $25^{\circ} \mathrm{C}$. The data were evaluated using volume distribution.

Zeta potential measurements of the blank and drug-loaded NPs in suspension diluted with deionized water were performed using a Zeta PALS (Brookhaven Instruments, Holtsville, NY). The zeta potential values were measured at the default parameters of dielectric constant, refractive index, and viscosity of water, with a disposable capillary cell with a volume of $1 \mathrm{~mL}$ at $25^{\circ} \mathrm{C} \pm 1^{\circ} \mathrm{C}$. All measurements were performed in triplicate.

\section{Determination of entrapment efficiency (EE) and drug loading (DL)}

The CUR content in the drug-loaded NPs was determined using the ultraviolet spectrophotometer at $425 \mathrm{~nm}$. The drug-loading content and drug entrapment efficiency were calculated based on the following formula: ${ }^{16}$

$$
\begin{gathered}
\mathrm{EE}=\frac{\text { Weight of drug in NPs }}{\text { Weight of the initial drug }} \times 100 \% \\
\text { DL content }=\frac{\text { Weight of drug in NPs }}{\text { Weight of NPs }} \times 100 \%
\end{gathered}
$$

\section{Differential scanning calorimetry (DSC) and FT-IR studies of CUR, CUR-free and -loaded NPs}

DSC analysis was performed to provide further information on the drug-copolymer relationship using a differential scanning calorimeter (Diamond TG/DTA, Perkin Elmer, Inc, Waltham, MA). Approximately $10 \mathrm{mg}$ of crude CUR powder or lyophilized NPs (with or without drug) was respectively sealed in the standard aluminum pan, purged in the calorimeter with pure dried nitrogen gas set at a flow rate of $10 \mathrm{~mL} / \mathrm{min}$, and heated at a constant rate of $10^{\circ} \mathrm{C} / \mathrm{min}$ from $0^{\circ} \mathrm{C}$ to $200^{\circ} \mathrm{C}$.

FT-IR spectra of native CUR and lyophilized NPs (with or without drug) were obtained by using potassium bromide pellets on the FT-IR spectrometer, over the range of $4000-400 \mathrm{~cm}^{-1}$.

\section{In vitro release of CUR-loaded NPs}

In vitro drug-release profile of CUR from drug-loaded NPs was carried out by the direct dispersion method as described in the literature. ${ }^{17-19}$ As a control, CUR was dissolved in a reported solution - containing $15 \% N, N$-dimethylacetamide (DMA), 45\% PEG400, and 40\% dextrose solution, with the concentration of dextrose at $5 \%$ - to form a CUR solution for injection. ${ }^{16}$ In vitro drug-release studies were done at $\mathrm{pH}$ 5.0. A known quantity of either the CUR-NP solution or control solution was placed in $30 \mathrm{~mL}$ of $10 \mathrm{mmol} / \mathrm{L}$ phosphate-buffered saline and this was divided into 30 Eppendorf tubes (ten sets of three tubes). The tubes were then incubated in a water bath shaker and oscillated at $100 \mathrm{r} / \mathrm{min}$ at $37^{\circ} \mathrm{C}$. Due to the low solubility of CUR in acidic conditions $(11 \mathrm{ng} / \mathrm{mL}$ at $\mathrm{pH} 5.0),{ }^{20}$ one set of tubes was taken out and centrifuged at $1200 \mathrm{r} / \mathrm{min}$ for 3 minutes to precipitate the released drug at definite time intervals, leaving the entrapped drug within NPs in the supernatant. The preciptate were dissolved in $4 \mathrm{~mL}$ ethanol and the amount of drug released was quantified spectrophotometerically at a wavelength of $425 \mathrm{~nm}$.

Wistar rats with body weights of $200 \pm 20 \mathrm{~g}$ were purchased from the Experimental Animal Center of Shandong University (Jinan City, Shandong Province, China). All animal experiments complied with the requirements of the National Act on the Use of Experimental Animals (China). Rats were fasted for 12 hours before the experiment with free access to water. They were randomly divided into two groups of five animals each.

The control solution, obtained from the section of in vitro release of CUR-loaded NPs and CUR-NP solution, were injected into the rats via the tail vein at a dose of $15 \mathrm{mg} / \mathrm{kg}$. At each time point, blood samples were collected via sinus venosus, immediately placed into heparinized tubes, and centrifuged at $3000 \mathrm{r} / \mathrm{min}$ for 10 minutes to obtain plasma.

The plasma samples were treated according to a previously reported method. ${ }^{16}$ CUR was then measured using reversed-phase high-performance liquid chromatography with a ultraviolet detector (Shimadzu LC-10ATVP, Kyoto, Japan). ${ }^{21}$ The mobile phase was composed of $52 \%$ acetonitrile and $48 \%$ citric buffer $(1 \% \mathrm{w} / \mathrm{v}$ citric acid solution adjusted to $\mathrm{pH} 3.0$ using concentrated sodium hydroxide solution). The flow rate was $1.0 \mathrm{~mL} / \mathrm{min}$ and the detection wavelength was at $425 \mathrm{~nm}$.

\section{Results and discussion Characterization of the structure of PCL-PEG-PCL}

PCL-PEG-PCL was successfully synthesized by ringopening copolymerization of PEG and $\varepsilon$-CL (Figure 2). 


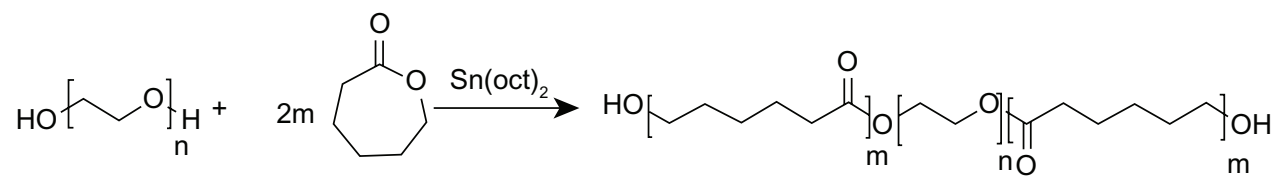

Figure 2 Synthesis of poly ( $\varepsilon$-Caprolactone)-poly (ethylene glycol)-poly ( $\varepsilon$-Caprolactone).

Triblock copolymers of different molecular weights were obtained by controlling the $\varepsilon$-CL:PEG ratio. Characteristics of the prepared copolymers are shown in Table 1.

FT-IR spectra shown in Figure 3. For PCL-PEG-PCL, the absorption band at $1725 \mathrm{~cm}^{-1}$ was attributed to the $\mathrm{C}=\mathrm{O}$ stretching vibrations of the ester carbonyl group. The peaks at 1107 and $1246 \mathrm{~cm}^{-1}$ were assigned to the characteristic $\mathrm{C}-\mathrm{O}-\mathrm{C}$ stretching vibration of repeated $-\mathrm{OCH}_{2} \mathrm{CH}_{2}$ units of PEG and the stretching vibrations of the $-\mathrm{COO}-$ bonds, respectively. The signals at 2944 and $2866 \mathrm{~cm}^{-1}$ correspond to the characteristic absorption of the $\mathrm{C}-\mathrm{H}$ stretching bonds of $-\mathrm{CH}_{2} \mathrm{CH}_{2}$, which are similar to those of $\varepsilon$-CL. The absorption peak at $3436 \mathrm{~cm}^{-1}$ shows that there was a terminal hydroxyl group in the PCL-PEG-PCL. These signals indicate that the PCL-PEG-PCL block copolymer was synthesized. ${ }^{22}$

To confirm the structure of the triblock copolymer, the ${ }^{1} \mathrm{H}-\mathrm{NMR}$ spectrum was recorded (Figure 4). The sharp single peak at $3.65 \mathrm{ppm}$ was attributed to the methylene protons of the PEG segments. The very weak peak at 4.23 ppm was identified as the methylene proton of the PEG end unit. In addition, peaks at $1.38,1.65,2.31$, and $4.06 \mathrm{ppm}$ were assigned to the methylene protons of $\mathrm{OCOCH}_{2} \mathrm{CH}_{2}-\mathbf{C H}_{2}-$ $\mathrm{CH}_{2} \mathrm{CH}_{2}, \mathrm{OCOCH}_{2}-\mathbf{C H}_{2}-\mathrm{CH}_{2}-\mathbf{C H}_{2}-\mathrm{CH}_{2}, \mathrm{OCO}-\mathbf{C H}_{2}-$ $\left(\mathrm{CH}_{2}\right)_{4}$, and $\mathrm{OCOCH}_{2} \mathrm{CH}_{2} \mathrm{CH}_{2} \mathrm{CH}_{2}-\mathbf{C H}_{2}$ in PCL units, respectively, being very similar to the reported spectrum. ${ }^{23}$

Table I Characteristics of nanoparticles (NPs)

\begin{tabular}{llllllll}
\hline NP & $\begin{array}{l}\text { PEG MW } \\
(\mathbf{g} / \mathbf{m o l})\end{array}$ & $\begin{array}{l}\text { Feed } \\
\text { ratio }^{\mathbf{a}}\end{array}$ & $\begin{array}{l}\text { MW } \\
\text { theo }^{\mathbf{b}}\end{array}$ & $\begin{array}{l}\text { MW } \\
\text { GPC }^{\mathbf{c}}\end{array}$ & PDI $^{\mathbf{d}}$ & $\begin{array}{l}\text { DL } \\
(\%)\end{array}$ & $\begin{array}{l}\text { EE } \\
(\%)\end{array}$ \\
\hline NP4I & 4000 & $\mathrm{I}: 0.5$ & 6000 & 5094 & 1.12 & 8.8 & 70.6 \\
NP42 & 4000 & $\mathrm{I}: 0.8$ & 7200 & 5544 & 1.33 & 10.7 & 85.5 \\
NP43 & 4000 & $\mathrm{I}: 1.3$ & 9200 & 18,050 & 2.54 & 11.8 & 94.3 \\
NP44 & 4000 & $\mathrm{I}: 2$ & 12,000 & 8212 & 1.44 & 10.9 & 87.4 \\
NP45 & 4000 & $\mathrm{I}: 3$ & 16,000 & 14,246 & 1.73 & 10.1 & 80.6 \\
NP6I & 6000 & $\mathrm{I}: 0.5$ & 9000 & 6132 & 1.19 & 2.7 & 21.9 \\
NP62 & 6000 & $\mathrm{I}: 0.8$ & 10,800 & 19,156 & 2.24 & 9.6 & 76.7 \\
NP63 & 6000 & $\mathrm{I}: 1.3$ & 13,800 & 19,367 & 2.61 & 11.9 & 95.5 \\
NP64 & 6000 & $\mathrm{I}: 2$ & 18,000 & 23,946 & 2.83 & 11.5 & 91.9 \\
NP65 & 6000 & $\mathrm{I}: 4$ & 30,000 & 24,812 & 1.52 & 4.3 & 34.4 \\
\hline
\end{tabular}

Notes: ${ }^{\mathrm{a} F e e d}$ weight ratio of poly (ethylene glycol) (PEG): $\varepsilon$-Caprolactone; btheoretical molecular weight (MW) based on feed weight ratio; ${ }^{\mathrm{MWW}}$ determined by gel permeation chromatography (GPC); d polydispersity index (PDI) determined by gel permeation chromatography (GPC).

Abbreviations: DL, drug loading; EE, entrapment efficiency.
All the FT-IR and ${ }^{1} \mathrm{H}-\mathrm{NMR}$ results indicated that the PCLPEG-PCL copolymer formed successfully.

Figure 5 shows the typical GPC curve of the NP63 copolymer. Only a single peak existed, indicating the monodistribution of macromolecular weight and absence of any homopolymer $\varepsilon$-CL or PEG, which also implies that no transesterification or backbiting reactions occurred during polymerization. ${ }^{24}$

\section{Characterization of NPs}

To develop an ideal formulation of higher DL or EE, two series of nanoparticulate CUR (based on PCL-PEG-PCL) were synthesized and the effects of the ratio of PCL and PEG on DL and EE were studied. The DL and EE of NP41-45 and NP61-65 are listed in Table 1. For both series of NPs with fixed PEG length, the DL and EE increased with the rise of feed weight ratio of PEG:E-CL (from 1:0.5 to 1:1.3). The maximum EE reached $94.3 \%$ and $95.5 \%$ for PEG-4000 and PEG-6000, respectively, when the ratio was 1:1.3. DL and EE decreased with the rise of feed weight ratio of PEG: $\varepsilon-C L$ from 1:2 to 1:3. Amphiphilic PCL-PEG-PCL copolymer formed NPs with a core-shell structure in water, where the core and shell were a hydrophobic PCL chain and a hydrophilic PEG chain, respectively. The hydrophobic drug could then be encapsulated into a hydrophobic core by means of the hydrophobic interaction of PCL and CUR and, with the inter-attraction between water and the hydrophilic shell of PEG, the drug was dissolved in water. ${ }^{25}$ The increase of the DL and EE of CUR in PCL-PEG-PCL may be caused by the strong hydrophobic interaction between the longer hydrophobic PCL block chain and hydrophobic drug. After DL, the size of the NPs increased (as shown in Figure 6 for NP43 and NP63). This result was expected because incorporation of CUR into the hydrophobic cores increased the volume of the NPs.

However, when the weight ratio changed from 1:1.3 to $1: 2$ and 1:3, the values of DL and EE reduced. This may be due to the poorer solubility of copolymers with longer PCL chains in an aqueous medium. Therefore, NP43 and NP63 were selected for in vitro release experiments.

The zeta potentials of blank NP43 and NP63 NPs were -4.8 and $-5.1 \mathrm{mV}$, respectively, whereas the zeta potentials of NP43 and NP63 CUR-NPs were -4.77 
A

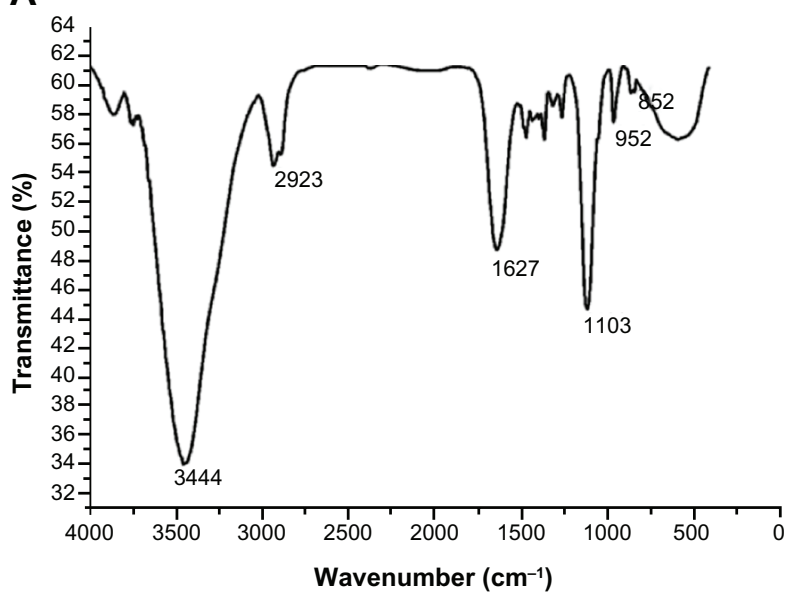

B

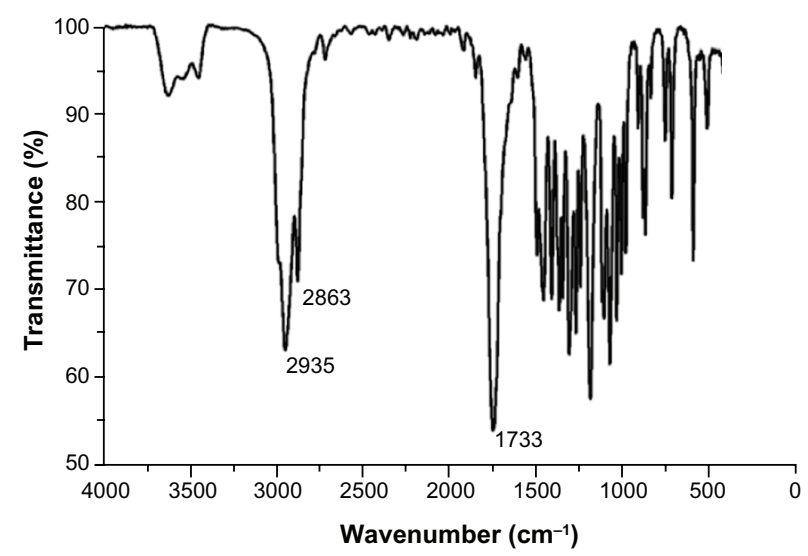

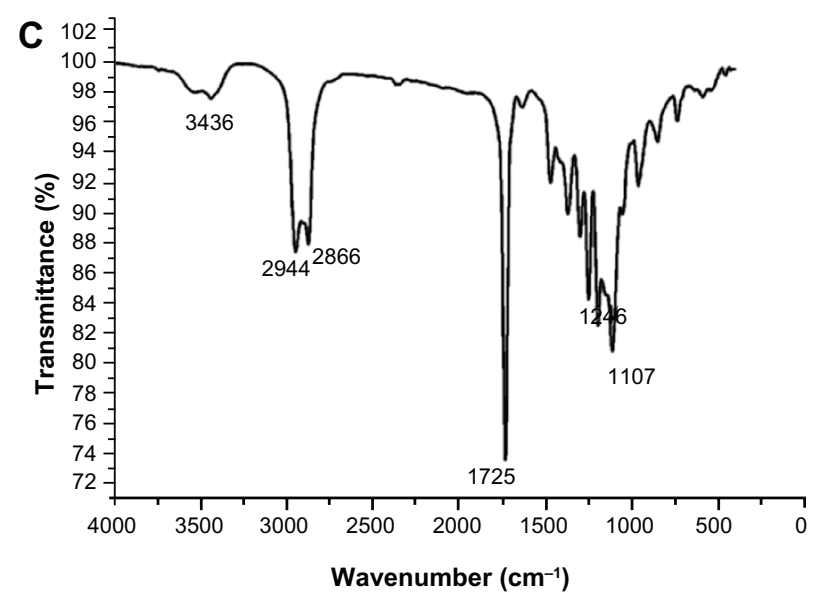

Figure 3 Fourier transform infrared spectra of poly (ethylene glycol) $(\mathbf{A}), \varepsilon$-Caprolactone (B) and poly ( $\varepsilon$-Caprolactone)-poly (ethylene glycol)-poly ( $\varepsilon$-Caprolactone) $(\mathbf{C})$.

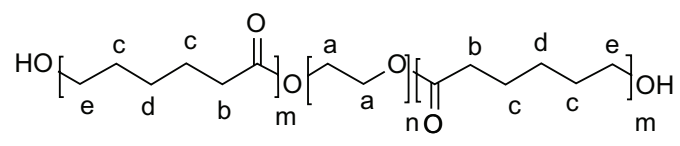

a

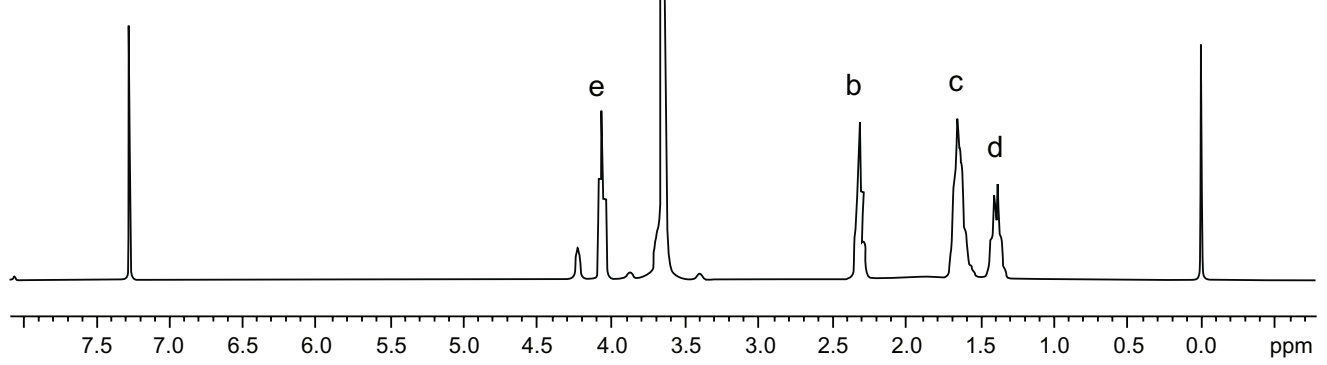

Figure 4 Proton nuclear magnetic resonance spectrum of poly ( $\varepsilon$-Caprolactone)-poly (ethylene glycol)-poly ( $\varepsilon$-Caprolactone). 


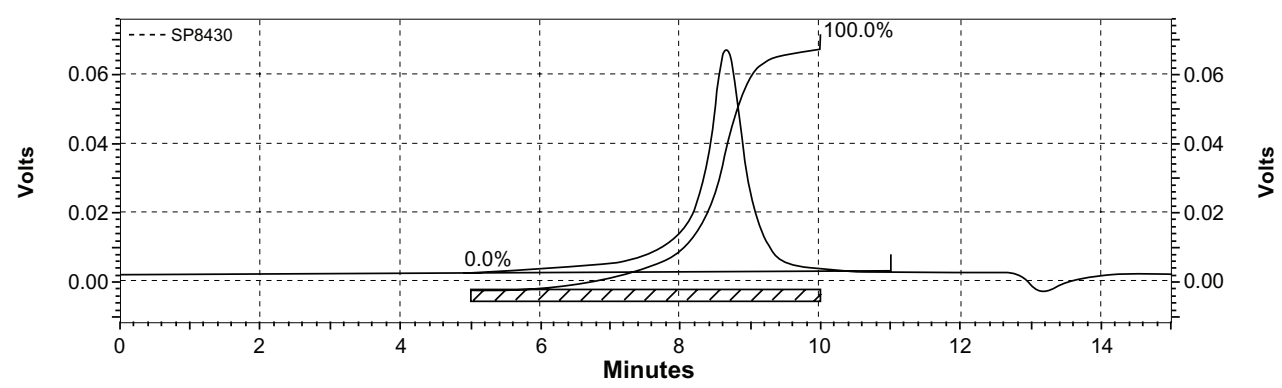

Figure $5 \mathrm{Gel}$ permeation chromatogram of poly ( $\varepsilon$-Caprolactone)-poly (ethylene glycol)-poly ( $\varepsilon$-Caprolactone).

and $-4.25 \mathrm{mV}$, respectively. The mean particle diameters of blank NP43 and NP63 NPs were 21.3 and $38.9 \mathrm{~nm}$, respectively, with a narrow monodispersed unimodal size distribution pattern (Figure 6). These were smaller than those of CUR-NPs for NP43 and NP63 (55.6 and $62.4 \mathrm{~nm}$, respectively). The TEM image (Figure 7) shows a discontinuous spherical outline.

To confirm drug presence in the nanoparticulate CUR formulation, FT-IR analysis was undertaken. Figure 8 shows the FT-IR spectra of native CUR, PCL-PEG-PCL, void NP63, and CUR-loaded NP63. The FT-IR spectrum of native CUR exhibited an absorption band at $3510 \mathrm{~cm}^{-1}$, which was attributed to the phenolic $\mathrm{O}-\mathrm{H}$ stretching vibration. ${ }^{26}$ In nanoparticulate CUR, a shift from 3510 to $3501 \mathrm{~cm}^{-1}$ was seen and the peak of $3501 \mathrm{~cm}^{-1}$ became wider, indicating
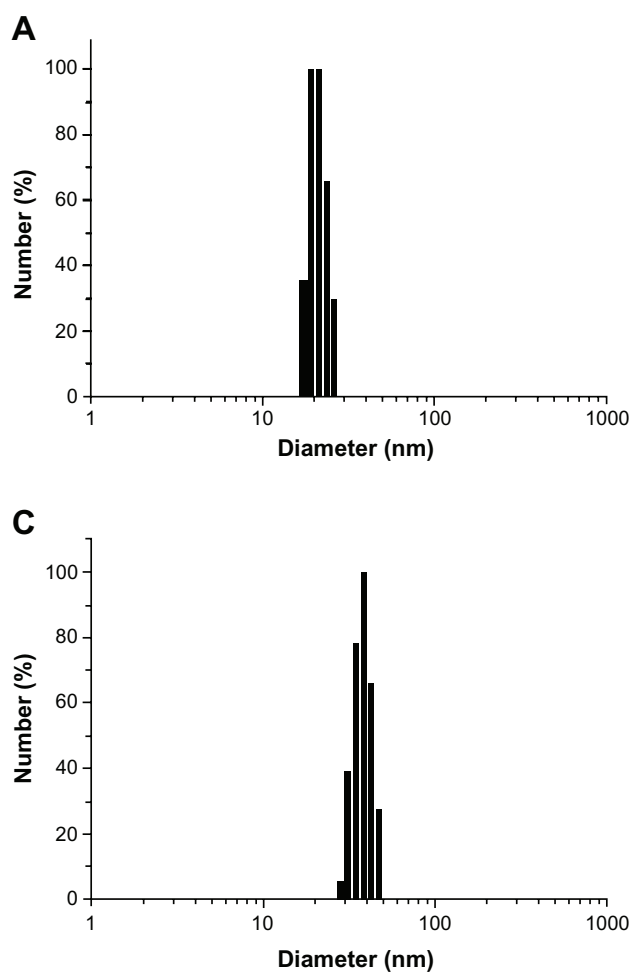

that hydrogen bonding was enhanced. The absorption peak of terminal hydroxyl of PCL-PEG-PCL at $3436 \mathrm{~cm}^{-1}$ in curve B changed into 3527 and $3501 \mathrm{~cm}^{-1}$ for void NPs and CUR-loaded NP63, respectively, further indicating that there was hydrogen bonding in PCL-PEG-PCL alone or PCL-PEGPCL with CUR. ${ }^{27}$

In curves $\mathrm{B}, \mathrm{C}$ and $\mathrm{D}$, the peaks at 1107 and $1246 \mathrm{~cm}^{-1}$ were assigned to the characteristic $\mathrm{C}-\mathrm{O}-\mathrm{C}$ stretching vibration of repeated $-\mathrm{OCH}_{2} \mathrm{CH}_{2}$ units of $\mathrm{PEG}$ and the $-\mathrm{COO}-$ bond-stretching vibrations, respectively. The signals at 2944 and $2866 \mathrm{~cm}^{-1}$ corresponded to the characteristic absorption of the $\mathrm{C}-\mathrm{H}$ stretching bonds of $-\mathrm{CH}_{2} \mathrm{CH}_{2}$, which were similar to those of $\varepsilon$-CL. Similarly, a weak peak at $1470 \mathrm{~cm}^{-1}$, observed in curves $\mathrm{B}, \mathrm{C}$ and $\mathrm{D}$, may be due to $-\mathrm{CH}_{2}$ bending vibration. The strong peak at $1725 \mathrm{~cm}^{-1}$ was due to $\mathrm{C}=\mathrm{O}$ adsorption.
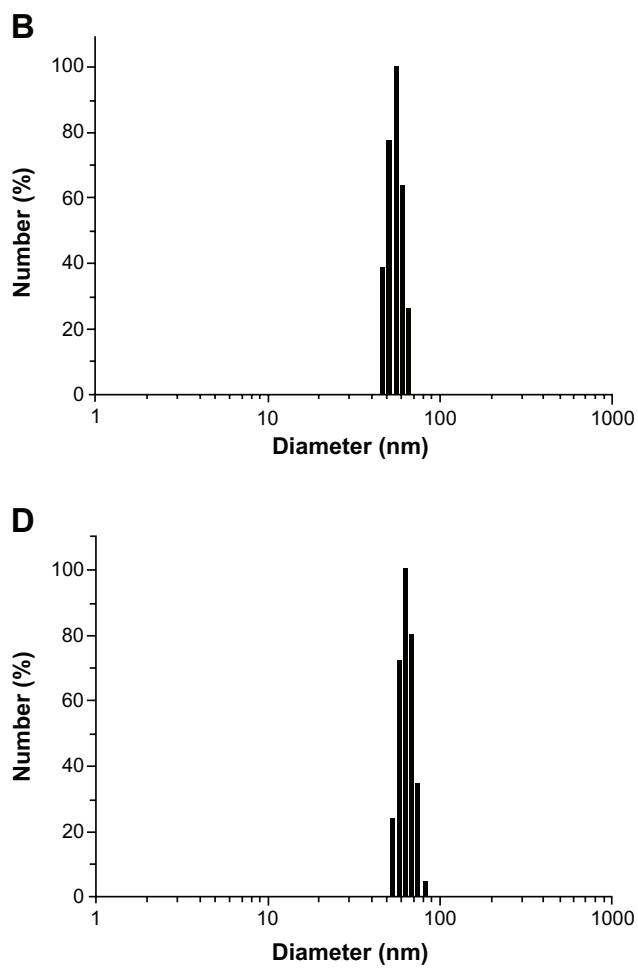

Figure 6 Particle size distribution of NP43 (blank (A) and curcumin-loaded (B)) and NP63 (blank (C) and curcumin-loaded (D)). 


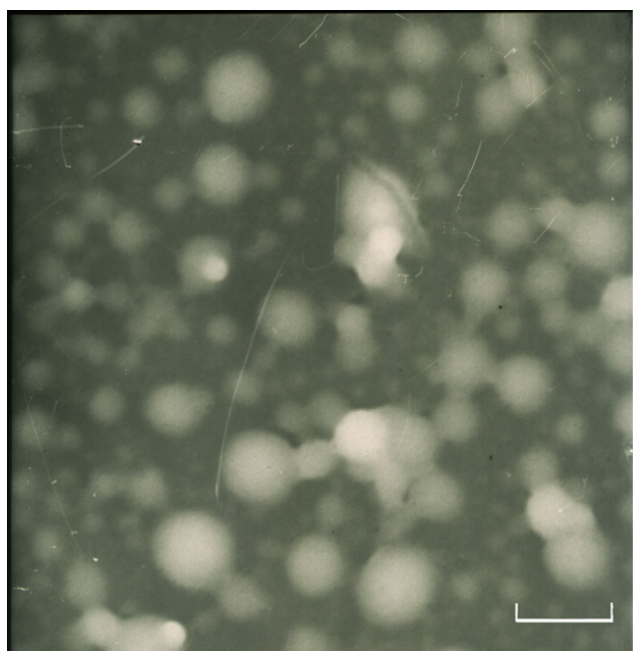

Figure 7 Transmission electron microscopy image of curcumin-loaded NP63. Notes: $72,000 \times$ magnification; scale bar is $100 \mathrm{~nm}$.

The signature peaks at 1627 and $1602 \mathrm{~cm}^{-1}$, found in native CUR but not in void NPs, were assumed to be due to $\mathrm{C}=\mathrm{C}$ double bonds and aromatic $\mathrm{C}=\mathrm{C}$ double bonds, respectively. Further, peaks similar to native CUR at 1627 and $1592 \mathrm{~cm}^{-1}$ were found in nanoparticulate CUR. All results indicated that CUR may be present in a dispersed condition in the nanoparticulate CUR formulation. ${ }^{27}$

In addition, the physical state of a drug in the polymeric matrix of NPs has been reported to influence the drug-release characteristics. ${ }^{28}$ DSC thermograms of native CUR, void NP63, and CUR-loaded NP63 are shown in Figure 9. The results show an endothermic peak of PCLPEG-PCL at a glass transition temperature of $45^{\circ} \mathrm{C}$. The endothermic peak of native CUR was found approximately at $175^{\circ} \mathrm{C}$, which was not observed in nanoparticulate CUR. Thus, it could be concluded that the CUR in the NPs was in an amorphous or disordered crystalline phase or in a solid solution state. ${ }^{28}$

\section{In vitro release of CUR-NPs}

The in vitro release behaviors of CUR solution and CUR-NPs in buffered solution (pH 5.0) are representatively shown in Figure 10. In a releasing medium of $\mathrm{pH} 5.0$, rapid release was observed in the controlled solution, with $97 \%$ of CUR released in 1 hour. The typical two-phase release profile was observed for CUR release from NP43 and NP63; that is, a relatively rapid release in the first stage followed by a sustained and slow release rate over a prolonged time of up to 96 hours. Overall, they showed a slower release than that of the control solution. At 96 hours, only $65.3 \%$ and $55 \%$ of the CUR was released from NP43 and NP63, respectively. It seems that the release rate of CUR from drug-loaded polymeric NPs decreased as the molecular weight of PCL-PEG-PCL in pH 5.0 buffers increased. The drug's release from amphiphilic copolymer NPs containing an ester group was due to hydrolysis
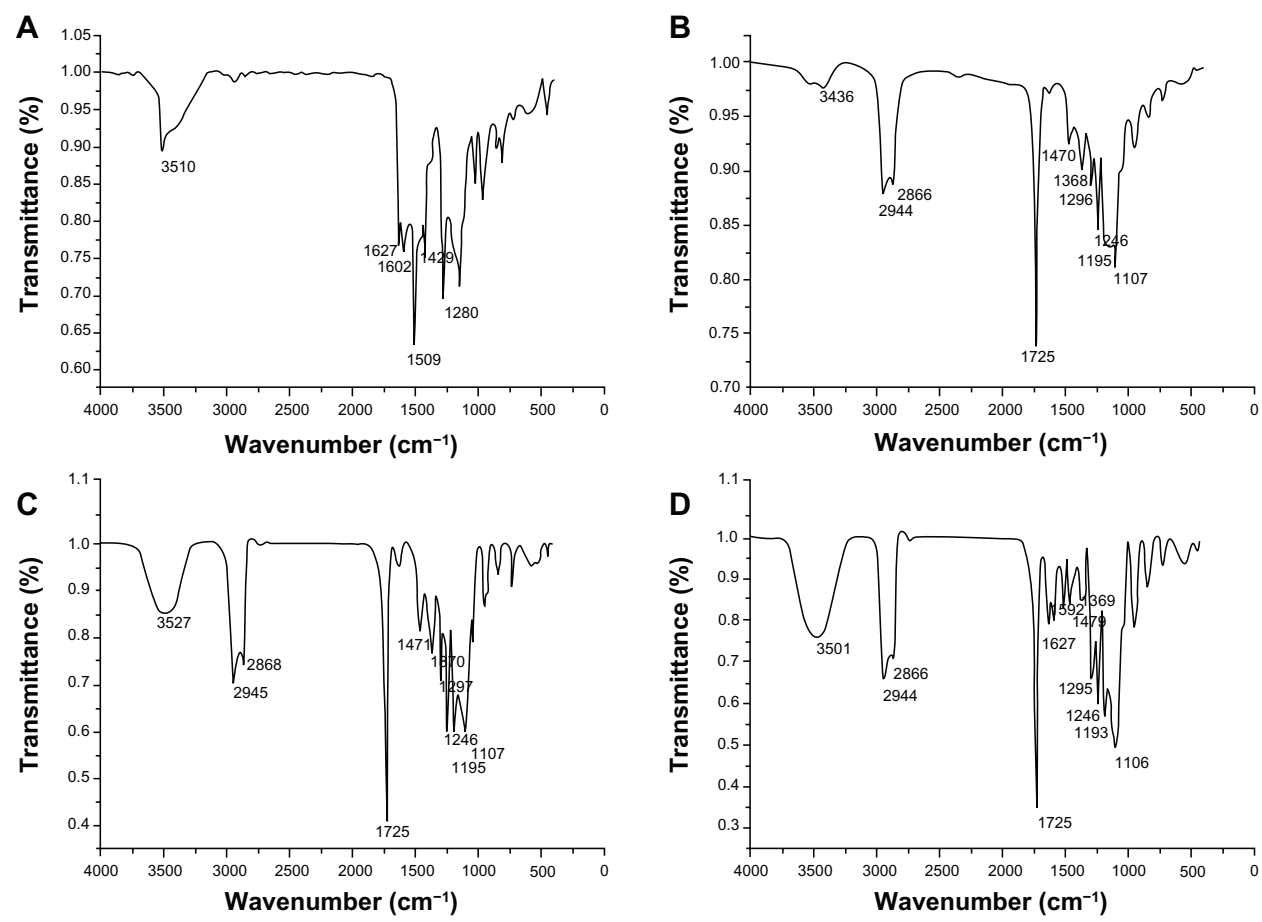

Figure 8 Fourier transform infrared spectra of native curcumin (A), poly ( $\varepsilon$-Caprolactone)-poly (ethylene glycol)-poly ( $\varepsilon$-Caprolactone) (B), void NP63 (C), and curcuminloaded NP63 (D). 


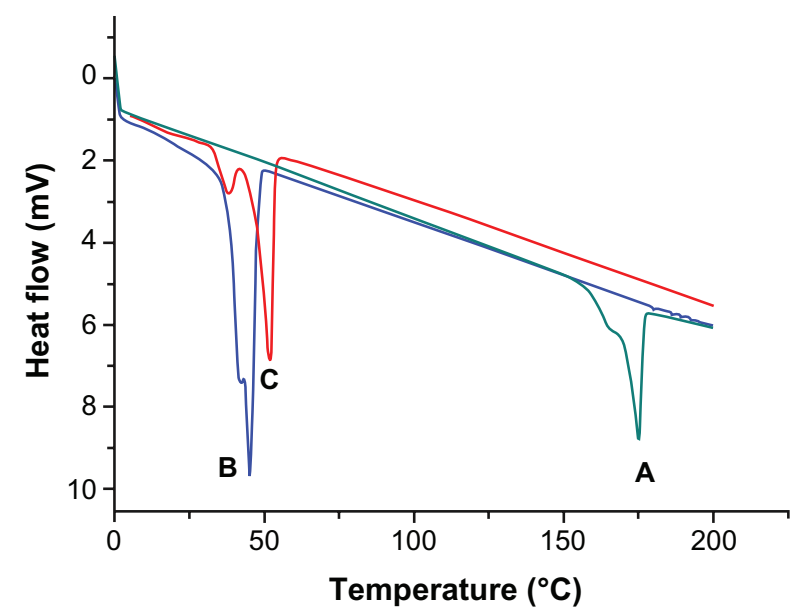

Figure 9 Differential scanning calorimetry thermograms of native curcumin (A), void NP63 (B), and curcumin-loaded NP63 (C).

disruption of the ester bonds and the drug's diffusion. The hydrolysis and drug's diffusion were controlled by the length of hydrophobic chain or hydrophilic chain, and $\mathrm{pH}$ or the temperature of medium. ${ }^{29-31}$ The slower release of NP63 at pH 5.0 may be mainly due to hydrolysis of ester groups. As the length of the hydrophobic ester chain in NP63 was longer than that in NP43, its hydrolysis was slower, which led to the slower release of CUR.

\section{Pharmacokinetics of CUR-NPs}

Since NP63 showed better EE, DL, and in vitro-release properties, we selected NP63 for pharmacokinetic research. The profiles of CUR concentration in plasma versus time are shown in Figure 11. It took longer to eliminate the CUR from NPs than from the control.

The pharmacokinetic parameters were calculated using the DAS 2.0(Mathematical Pharmacology Professional Committee

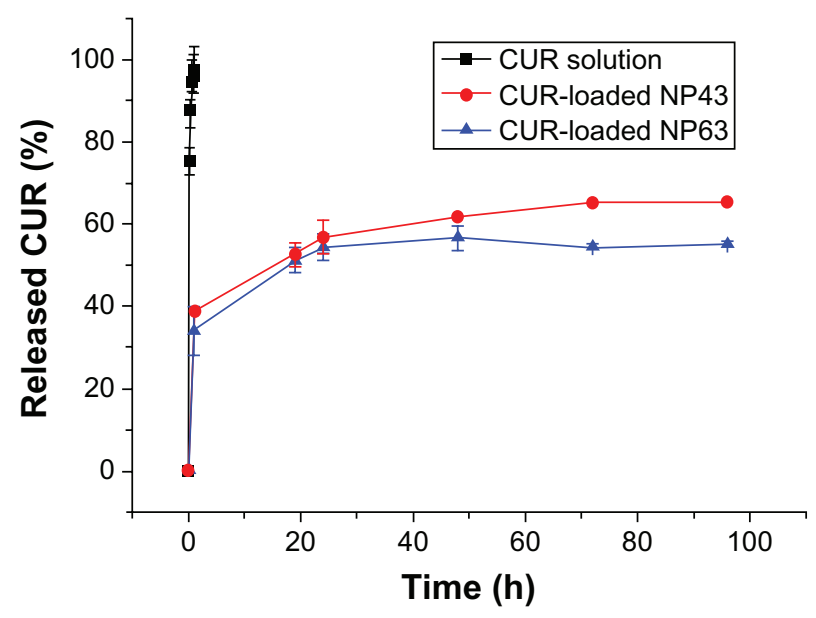

Figure 10 In vitro curcumin (CUR)-release profiles from two NPs (NP43 and NP63) at $\mathrm{pH} 5.0$ at $37^{\circ} \mathrm{C}$.

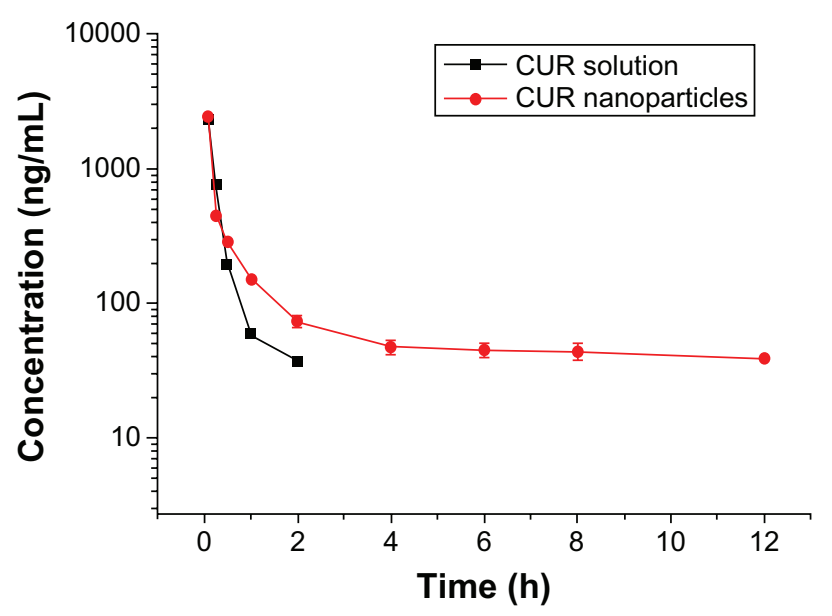

Figure I I Concentration profile of curcumin (CUR) in plasma versus time after intravenous injection of CUR solution or CUR nanoparticles.

of China, Shanghai, China) practical pharmacokinetics program for noncompartmental models. The pharmacokinetic parameters, such as area under the concentration-time curve $(\mathrm{AUC})_{(0-\infty)}$, mean residence time (MRT), total clearance (CL), steady-state volume of distribution (Vss) and biological halflife $\left(\mathrm{t}_{1 / 2}\right)$, are reported in Table 2 .

As shown in Table 2, the pharmacokinetic parameters of CUR-NPs in rats showed significant change in comparison to those of the CUR solution. NPs provided higher AUC ${ }_{(0-\infty)}$ (4.15-fold) and MRT (178.99-fold) compared with the CUR solution. The CUR-NPs also had decreased CL compared with the CUR solution. The extended MRT and decreased CL indicated that CUR-NPs could prolong the acting time of CUR in vivo. The Vss of the drug encapsulated in NPs $(0.216 \mathrm{~L} / \mathrm{kg})$ was significantly greater than that in the CUR solution $(0.004 \mathrm{~L} / \mathrm{kg})$. This result indicates that CUR-NPs could extend the retention time and delay clearance of CUR. The more NPs circulate in blood for a longer period, the greater their accumulation in a tumor may be. The ratio of $\mathrm{AUC}_{\mathrm{NP}(0-\infty)}$ to $\mathrm{AUC}_{\mathrm{sol}(0-\infty)}$ was 4.15, indicating that the CURNPs provided a higher systemic exposure of CUR than the CUR solution.

Table 2 The pharmacokinetic parameters of curcumin (CUR) solution and CUR nanoparticles (NPs) $(n=5)$

\begin{tabular}{lll}
\hline Parameters & CUR solution & CUR NPs \\
\hline $\operatorname{AUC}_{(0-\infty)}(\mathrm{mg} / \mathrm{L} * \mathrm{~h})$ & 668.415 & 2772.523 \\
$\mathrm{MRT}_{(0-\infty)}(\mathrm{h})$ & 0.168 & 30.07 \\
$\mathrm{t}_{\mathrm{l} / 2}(\mathrm{~h})$ & 0.135 & 27.618 \\
$\mathrm{Vss}(\mathrm{L} / \mathrm{kg})$ & 0.004 & 0.216 \\
$\mathrm{CL}(\mathrm{L} / \mathrm{h} / \mathrm{kg})$ & 0.022 & 0.005 \\
\hline
\end{tabular}

Abbreviations: AUC, area under the concentration-time curve; $\mathrm{CL}$, total clearance; MRT, mean residence time; $t_{1 / 2}$, biological half-life; Vss, steady-state volume of distribution. 


\section{Conclusion}

This study explored the effect of the length of hydrophilic and hydrophobic chains on the encapsulation of hydrophilic CUR to obtain an appropriate sustained-release property for CUR in vitro. The results suggest that CUR-loaded NP63 is a suitable candidate for the in vivo delivery of CUR to rats via intravenous injection. The entrapment of CUR within a nanoparticulate carrier noticeably improved the pharmacokinetic parameters of CUR, including a marked increase in the MRT and $\mathrm{AUC}_{(0-\infty)}$ and substantial decreases in CL and Vss. Therefore, this NP formulation could effectively prolong the retention of CUR in the systemic circulation and hopefully improve the therapeutic index of CUR in the future.

\section{Acknowledgments}

This work was supported by a research grant from the Shandong Excellent Young Scientist Award Fund (BS2011CL006), People's Republic of China. It was also supported by a research grant from the Scientific Research Fund of the University of Jinan (XKY0910), People's Republic of China.

\section{Disclosure}

The authors report no conflicts of interest in this work.

\section{References}

1. Maheshwari RK, Singh AK, Gaddipati J, Srimal RC. Multiple biological activities of curcumin: a short review. Life Sci. 2006;78(18): 2081-2087.

2. Ono K, Hasegawa K, Naiki H, Yamada M. Curcumin has potent antiamyloidogenic effects for Alzheimer's beta-amyloid fibrils in vitro. J Neurosci Res. 2004;75(6):742-750.

3. Aggarwal BB, Kumar A, Bharti AC. Anticancer potential of curcumin: preclinical and clinical studies. Anticancer Res. 2003;23(1A):363-398.

4. Hatcher H, Planalp R, Cho J, Torti FM, Torti SV. Curcumin: from ancient medicine to current clinical trials. Cell Mol Life Sci. 2008;65(11):1631-1652.

5. Letchford K, Liggins R, Burt H. Solubilization of hydrophobic drugs by methoxy poly(ethylene glycol)-block-polycaprolactone diblock copolymer micelles: theoretical and experimental data and correlations. J Pharm Sci. 2008;97(3):1179-1190.

6. Anand P, Kunnumakkara AB, Newman RA, Aggarwal BB. Bioavailability of curcumin: problems and promises. Mol Pharm. 2007;4(6):807-818.

7. Avgoustakis K, Beletsi A, Panagi Z, et al. Effect of copolymer composition on the physicochemical characteristics, in vitro stability, and biodistribution of PLGA-mPEG nanoparticles. Int J Pharm. 2003;259(1-2):115-127.

8. Stella B, Arpicco S, Peracchia MT, et al. Design of folic acid-conjugated nanoparticles for drug targeting. J Pharm Sci. 2000;89(11):1452-1464

9. Shukla S, Wu G, Chatterjee M, et al. Synthesis and biological evaluation of folate receptor-targeted boronated PAMAM dendrimers as potential agents for neutron capture therapy. Bioconjug Chem. 2003;14(1):158-167.

10. Wang C, Cheng L, Liu Z. Drug delivery with upconversion nanoparticles for multi-functional targeted cancer cell imaging and therapy. Biomaterials. 2011;32(4):1110-1120.

11. Zhang Y, Zhuo RX. Synthesis and in vitro drug release behavior of amphiphilic triblock copolymer nanoparticles based on poly (ethylene glycol) and polycaprolactone. Biomaterials. 2005;26(33):6736-6742.
12. Liu D, Wang L, Liu Z, Zhang C, Zhang N. Preparation, characterization, and in vitro evaluation of docetaxel-loaded poly(lactic acid)poly(ethylene glycol) nanoparticles for parenteral drug delivery. J Biomed Nanotechnol. 2010;6(6):675-682.

13. Pirollo KF, Chang EH. Does a targeting ligand influence nanoparticle tumor localization or uptake? Trends Biotechnol. 2008;26(10):552-558.

14. Huang MJ, Gou ML, Qian ZY, et al. One-step preparation of poly(epsiloncaprolactone)-poly(ethylene glycol)-poly(epsilon-caprolactone) nanoparticles for plasmid DNA delivery. J Biomed Mater Res A. 2008;86(4):979-986.

15. Moon T, Pellinen D, Purcell E, Seymour J, Kipke D. Local drug delivery system for dynamic control of neural environment using parylene-based microelectrodes. IFMBE Proc. 2007;14(23):3542-3545.

16. Song Z, Feng R, Sun M, et al. Curcumin-loaded PLGA-PEG-PLGA triblock copolymeric micelles: Preparation, pharmacokinetics and distribution in vivo. J Colloid Interface Sci. 2011;354(1):116-123.

17. Bisht S, Feldmann G, Soni S, et al. Polymeric nanoparticle-encapsulated curcumin ("nanocurcumin"): a novel strategy for human cancer therapy. J Nanobiotechnology. 2007;5:3.

18. Anitha A, Deepagan VG, Divya Rani VV, Menon D, Nair SV, Jayakumar R. Preparation, characterization, in vitro drug release and biological studies of curcumin loaded dextran sulphate-chitosan nanoparticles. Carbohydr Polym. 2011;84(3):1158-1164.

19. AnithaA, Maya S, Deepa N, et al. Efficient water soluble O-carboxymethyl chitosan nanocarrier for the delivery of curcumin to cancer cells. Carbohydr Polym. 2011;83(2):452-461.

20. Tonnesen HH, Másson M, Loftsson T. Studies of curcumin and curcuminoids. XXVII. Cyclodextrin complexation: solubility, chemical and photochemical stability. Int J Pharm. 2002;244(1-2):127-135.

21. Ma Z, Shayeganpour A, Brocks DR, Lavasanifar A, Samuel J. Highperformance liquid chromatography analysis of curcumin in rat plasma: application to pharmacokinetics of polymeric micellar formulation of curcumin. Biomed Chromatogr. 2007;21(5):546-552.

22. Liu CB, Gong CY, Huang MJ, et al. Thermoreversible gel-sol behavior of biodegradable PCL-PEG-PCL triblock copolymer in aqueous solutions. J Biomed Mater Res B Appl Biomater. 2008;84(1):165-175.

23. Jia W, GuY, Gou M, et al. Preparation of biodegradable polycaprolactone/ poly (ethylene glycol)/polycaprolactone (PCEC) nanoparticles. Drug Deliv. 2008;15(7):409-416.

24. Zhou S, Deng X, Yang H. Biodegradable poly(epsilon-caprolactone)poly(ethylene glycol) block copolymers: characterization and their use as drug carriers for a controlled delivery system. Biomaterials. 2003;24(20):3563-3570.

25. Hu Y, Jiang X, Ding Y, et al. Preparation and drug release behaviors of nimodipine-loaded poly(caprolactone)-poly(ethylene oxide)polylactide amphiphilic copolymer nanoparticles. Biomaterials. 2003;24(13):2395-2404.

26. Yallapu MM, Jaggi M, Chauhan SC. beta-Cyclodextrin-curcumin selfassembly enhances curcumin delivery in prostate cancer cells. Colloids Surf B Biointerfaces. 2010;79(1):113-125.

27. Mohanty C, Sahoo SK. The in vitro stability and in vivo pharmacokinetics of curcumin prepared as an aqueous nanoparticulate formulation. Biomaterials. 2010;31(25):6597-6611.

28. Zhang Y, Tang L, Sun L, et al. A novel paclitaxel-loaded poly( $\varepsilon$ caprolactone)/Poloxamer 188 blend nanoparticle overcoming multidrug resistance for cancer treatment. Acta Biomater. 2010;6(6):2045-2052.

29. Xu B, Yuan JF, Ding T, Gao QY. Amphiphilic biodegradable poly( $\varepsilon$ caprolactone)-poly(ethylene glycol)-poly( $\varepsilon$-caprolactone) triblock copolymers: synthesis, characterization and their use as drug carriers for folic acid. Polymer Bulletin. 2010;64(6):537-551.

30. Hu Y, Xie J, Tong YW, Wang CH. Effect of PEG conformation and particle size on the cellular uptake efficiency of nanoparticles with the HepG2 cells. J Control Release. 2007;118(1):7-17.

31. Zhang L, Hu Y, Jiang X, Yang C, Lu W, Yang YH. Camptothecin derivative-loaded poly(caprolactone-co-lactide)-b-PEG-b-poly(caprolactoneco-lactide) nanoparticles and their biodistribution in mice. J Control Release. 2004;96(1):135-148. 


\section{Publish your work in this journal}

The International Journal of Nanomedicine is an international, peerreviewed journal focusing on the application of nanotechnology in diagnostics, therapeutics, and drug delivery systems throughout the biomedical field. This journal is indexed on PubMed Central, MedLine, CAS, SciSearch ${ }^{\circledR}$, Current Contents ${ }^{\circledR} /$ Clinical Medicine,

Journal Citation Reports/Science Edition, EMBase, Scopus and the Elsevier Bibliographic databases. The manuscript management system is completely online and includes a very quick and fair peer-review system, which is all easy to use. Visit http://www.dovepress.com/ testimonials.php to read real quotes from published authors.

Submit your manuscript here: http://www.dovepress.com/international-journal-of-nanomedicine-journal 